\title{
Waveguide Fabrication In Fused Silica Using Tightly Focused Femtosecond Laser Pulses
}

\author{
J.W. Chan, T.R. Huser, S.H. Risbud, D.M. Krol
}

This article was submitted to The Society of Photo-Optical Instrumentation Engineers Proceedings

Photonics West 2002, San jose, CA., January 19-25, 2002

January 7, 2002 


\section{DISCLAIMER}

This document was prepared as an account of work sponsored by an agency of the United States Government. Neither the United States Government nor the University of California nor any of their employees, makes any warranty, express or implied, or assumes any legal liability or responsibility for the accuracy, completeness, or usefulness of any information, apparatus, product, or process disclosed, or represents that its use would not infringe privately owned rights. Reference herein to any specific commercial product, process, or service by trade name, trademark, manufacturer, or otherwise, does not necessarily constitute or imply its endorsement, recommendation, or favoring by the United States Government or the University of California. The views and opinions of authors expressed herein do not necessarily state or reflect those of the United States Government or the University of California, and shall not be used for advertising or product endorsement purposes.

This is a preprint of a paper intended for publication in a journal or proceedings. Since changes may be made before publication, this preprint is made available with the understanding that it will not be cited or reproduced without the permission of the author.

This report has been reproduced directly from the best available copy.

Available to DOE and DOE contractors from the

Office of Scientific and Technical Information

P.O. Box 62, Oak Ridge, TN 37831

Prices available from (423) 576-8401

http:/ / apollo.osti.gov/bridge/

Available to the public from the National Technical Information Service

U.S. Department of Commerce 5285 Port Royal Rd., Springfield, VA 22161

http://www.ntis.gov/

OR

Lawrence Livermore National Laboratory Technical Information Department's Digital Library http://www.llnl.gov/tid/Library.html 


\title{
Waveguide fabrication in fused silica using tightly focused femtosecond laser pulses
}

\author{
James W. Chan ${ }^{1,2}$, Thomas R. Huser ${ }^{1}$, Subhash H. Risbud ${ }^{2}$, Denise M. Krol ${ }^{1,3}$ \\ 1. Lawrence Livermore National Laboratory, Livermore, CA 94550 \\ 2. Department of Chemical Engineering and Materials Science, University of California at Davis, \\ Davis, CA 95616 \\ 3. Department of Applied Science-Livermore, University of California at Davis, Livermore, CA \\ 94550
}

\begin{abstract}
Refractive index changes have been induced inside bulk fused silica by using femtosecond (fs) laser pulses tightly focused inside the material. Waveguides have been fabricated inside the glass by scanning the glass with respect to the focal point of the laser beam. The refractive index change is estimated to be $\sim 10^{-4}$. Other more complex three-

dimensional structures have also been fabricated (curved waveguides, splitters, and interferometers). We also report on fluorescence spectroscopy of the fs-modified fused silica using a confocal microscopy setup. Using a $488 \mathrm{~nm}$ excitation source, a fluorescence at $630 \mathrm{~nm}$ is observed from the modified glass, which is attributed to the presence of non-bridging oxygen hole center (NBOHC) defects created by the fs pulses. The fluorescence decays with prolonged exposure to the $488 \mathrm{~nm}$ light, indicating that the defects are being photobleached by the excitation light.
\end{abstract}

Keywords: femtosecond lasers, fused silica, laser microfabrication, optical device fabrication, confocal microscopy

\section{INTRODUCTION}

The need for optical switches, modulators, amplifiers, and other components for optical communication has spawned tremendous interest in the fabrication of glass-based integrated optical devices in recent years. Waveguides, the main structures in an integrated optical device, have been traditionally fabricated in glass using a variety of techniques such as ion exchange, sputtering, and chemical vapor deposition. Although the ion exchange technique is the most popular, the process only allows fabrication of the waveguide at or near the surface of the glass substrate. Also, more complex three dimensional structures are more difficult to fabricate inside the glass using this technique.

In recent years, femtosecond laser micromachining for the purpose of fabricating optical devices and components inside bulk glasses has been demonstrated. ${ }^{1-4} \mathrm{~A}$ variety of devices (waveguides, splitters, directional couplers, optical data storage devices, gratings) have been fabricated in different types of materials. The fabrication process involves focusing near-infrared fs laser pulses inside a bulk transparent material to induce refractive index changes at the focal point of the laser beam. When the glass is translated with respect to the focal point, complex three-dimensional structures can be fabricated inside the bulk glass substrate.

The intensity achieved in the focal volume is high enough for absorption of the near-infrared fs laser light through multiphoton absorption and avalanche ionization, leading to plasma formation. ${ }^{5,6}$ It has been proposed ${ }^{4}$ that plasma formation within the focal region introduces shock wave propagation inside the glass, as the temperature increases within a confined volume of material. The result of the perturbation is both a visual structural change and a change in the optical properties (i.e. refractive index) within a micron-sized region inside the glass.

In a recent study ${ }^{7}$, we have shown that a change in the structural network of fused silica occurs upon irradiation with tightly focused fs laser pulses. A confocal microscopy setup was used to locally probe the fs-modified material in the 
bulk glass for changes in the Raman scattering signals. The Raman changes indicated modification of the glass network, which is associated with densification of the glass. The densification is, at least, partly responsible for the induced refractive index change observed in the material. Aside from this change, we also observed a broad fluorescence when the modified glass was exposed to $488 \mathrm{~nm}$ excitation light. ${ }^{8}$ This fluorescence is known to be associated with nonbridging oxygen hole center (NBOHC) defects in fused silica. ${ }^{9}$

In this paper, we report on the fabrication and characterization of a variety of complex three-dimensional structures inside fused silica, which have important practical applications for the telecommunications industry. We also investigate, using a confocal fluorescence microscopy setup, the formation of the color center defects produced in fused silica by fs laser pulses and the behavior of the defects upon exposure to cw laser light.

\section{EXPERIMENTAL PROCEDURE}

\subsection{Optical device fabrication}

The experimental setup for fabrication of straight waveguides and other devices is illustrated in Figure 1. The fs laser beam $(800 \mathrm{~nm}, 130 \mathrm{fs}, 1 \mu \mathrm{J}, 1 \mathrm{kHz})$ was directed through a 10x $(\mathrm{NA}=0.25)$ objective using a pellicle beamsplitter and focused into polished fused silica cubes that were placed on a long-travel motorized translation stage with automated motion along the $\mathrm{x}$ - and $\mathrm{y}$-axes. For coupling of light into the fabricated devices, a He-Ne cw laser at $633 \mathrm{~nm}$ was directed through the same 10x objective. A second objective (20x) was used to image the near field profile of the coupled light through the device onto a CCD camera (\#2) connected to a computer. White light images of both the front and back planes of the fabricated device were captured using the two objectives and the two CCD cameras (\#1 and \#2).

fs beam

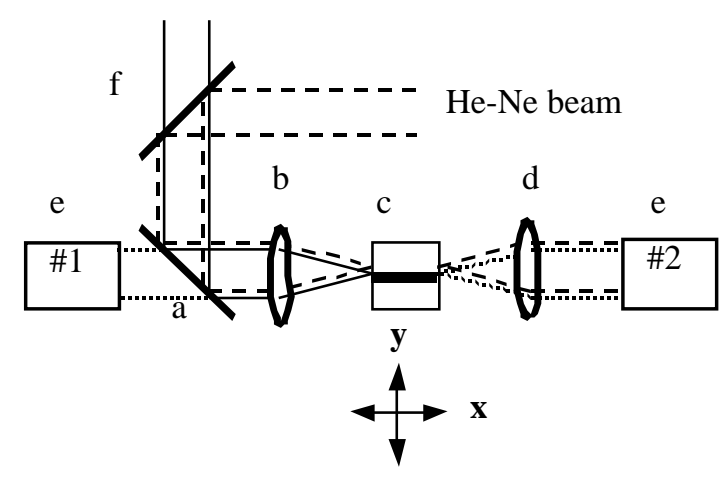

Fig. 1.: Setup for waveguide/device fabrication a- pellicle beamsplitter, b- 10x objective c- fused silica cube, d- $20 x$ objective, e - CCD cameras connected to computer, f - flip mirror

For the fabrication of the straight waveguide, the fused silica sample was scanned along the beam axis one pass at a rate of $20 \mu \mathrm{m} / \mathrm{s}$. The total scan length (and total waveguide length) of $\sim 5 \mathrm{~mm}$ was limited by the working distance of the 10x objective. We made sure that the focal spot did not scan across the surface of the samples on either side to eliminate the possibility of surface damage. The other devices (splitters, curved waveguides, interferometers) were fabricated by scanning the glass in both the $\mathrm{x}$ - and $\mathrm{y}$ - direction to "write" the appropriate structure, all the time maintaining the vectorial velocity at $20 \mu \mathrm{m} / \mathrm{s}$. 


\subsection{Confocal microscopy setup}

The confocal microscopy setup used to perform fluorescence spectroscopy on the samples is shown in Figure 2. A 15 $\mathrm{mW} \mathrm{cw}$ Ar ion laser at $488 \mathrm{~nm}$ was used as the excitation source. Both the fs beam and the excitation beam were focused into the sample using a 50x (N.A. $=0.55$ ) microscope objective (Nikon ELWD). The sample was placed on a computer controlled XYZ stage. The $488 \mathrm{~nm}$ excitation beam was filtered from the fluorescence signals using the dichroic beam splitter and a holographic notch filter. The $50 \mu \mathrm{m}$ diameter pinhole was used to ensure that only fluorescence signals from the focal volume of the objective were delivered to the spectrometer and liquid nitrogen cooled CCD camera (LN-CCD, Princeton Instruments). A grating with 300 grooves $/ \mathrm{mm}$ was used inside the spectrometer. A CCD camera was used to capture images of the fs-modified structures inside the glass using white light illumination.

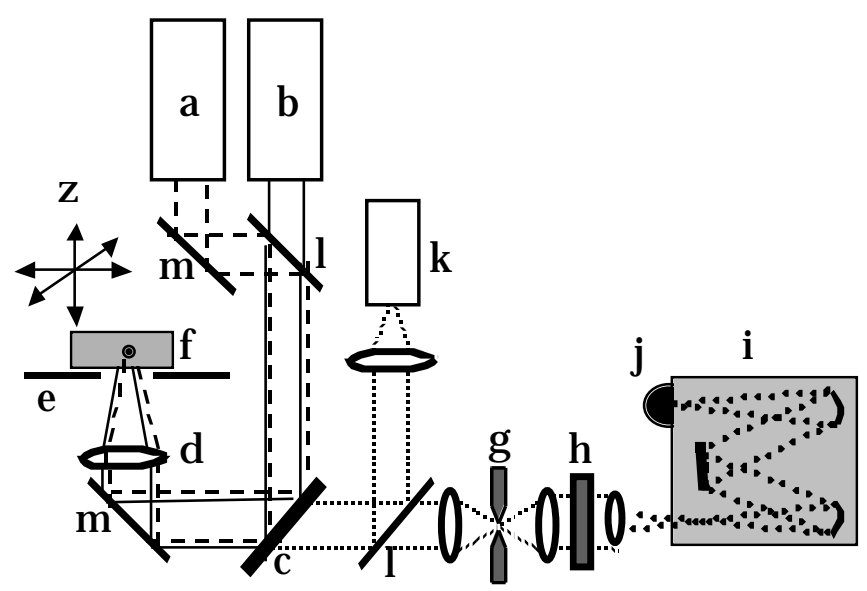

Fig. 2.: Confocal microscopy setup used to collect fluorescence spectra (a - fs laser system, b- $488 \mathrm{~nm}$ cw laser, $\mathrm{c}$ - dichroic beamsplitter, $\mathrm{d}$ - microscope objective, e- XYZ translation stage, $\mathrm{f}$ - glass, gspatial filter, h- $488 \mathrm{~nm}$ notch filter, $\mathrm{i}$ - spectrometer, $\mathrm{j}$ - LN cooled CCD camera, $k-C C D$ camera 1 flip mirrors)

In our experiments, we used fs pulse energies ranging from 0.1 to $2 \mu \mathrm{J}$. Using a knife edge technique, the diameter of the laser spot at the focal point of the objective was measured to be $2 \mu \mathrm{m}$. Fluences per pulse at the focal point ranged from $5-200 \mathrm{~J} \mathrm{~cm}^{-2}$. The laser beam was focused $\sim 700 \mu \mathrm{m}$ inside the fused silica samples which were placed on the XYZ stage. Large three-dimensional modified structures were formed inside the glass. The region consists of three 100 $\mathrm{x} 100 \mu \mathrm{m}^{2}$ planes spaced $2 \mu \mathrm{m}$ apart in the vertical direction (z). Each plane is made by scanning $128100 \mu \mathrm{m}$ lines spaced $0.8 \mu \mathrm{m}$ apart at a rate of $40 \mu \mathrm{m} / \mathrm{s}$. The relatively larger region is created to ensure that the Ar excitation beam will only probe the fs-modified glass, thereby circumventing any issues concerning how good the Ar beam overlaps the fs beam, particularly in the direction of the beam propagation (z-direction in Figure 2). The excitation beam is focused in the center of this region to probe for fluorescence signals.

\section{RESULTS AND DISCUSSION}

\subsection{Fabrication and characterization of waveguide structures inside fused silica}

\subsubsection{Waveguides}

Waveguides supporting the fundamental mode have been fabricated in fused silica by using femtosecond pulse energies ranging from 0.8 to $1.6 \mu \mathrm{J}$ (Figure 3a). Using white light microscopy, these waveguides looked smooth and continuous. Higher femtosecond pulse energies resulted in the formation of damage lines, which appeared rough and discontinuous. 
The diameter of the modified region is $\sim 9 \mu \mathrm{m}$ (Figure $3 \mathrm{~b}$ ), with the near field pattern at $633 \mathrm{~nm}$ through the waveguide (Figure 3c) having a nearly Gaussian mode profile. In order to estimate the refractive index change associated with fs modification of the glass, we measured the numerical aperture (NA) of the waveguide by analyzing the cone of the 633 $\mathrm{nm}$ coupled light exiting the waveguide. Assuming a step-index profile, the numerical aperture was then related to the refractive index change by the equation NA $\sqrt{2 n \Delta n}$ for small $\Delta \mathrm{n}$. The refractive index change was roughly $0.07 \mathrm{x}$ $10^{-3}$ with an error of $\sim 17 \%$. The magnitudes of these values are consistent with previously published studies. ${ }^{2}$

(a)
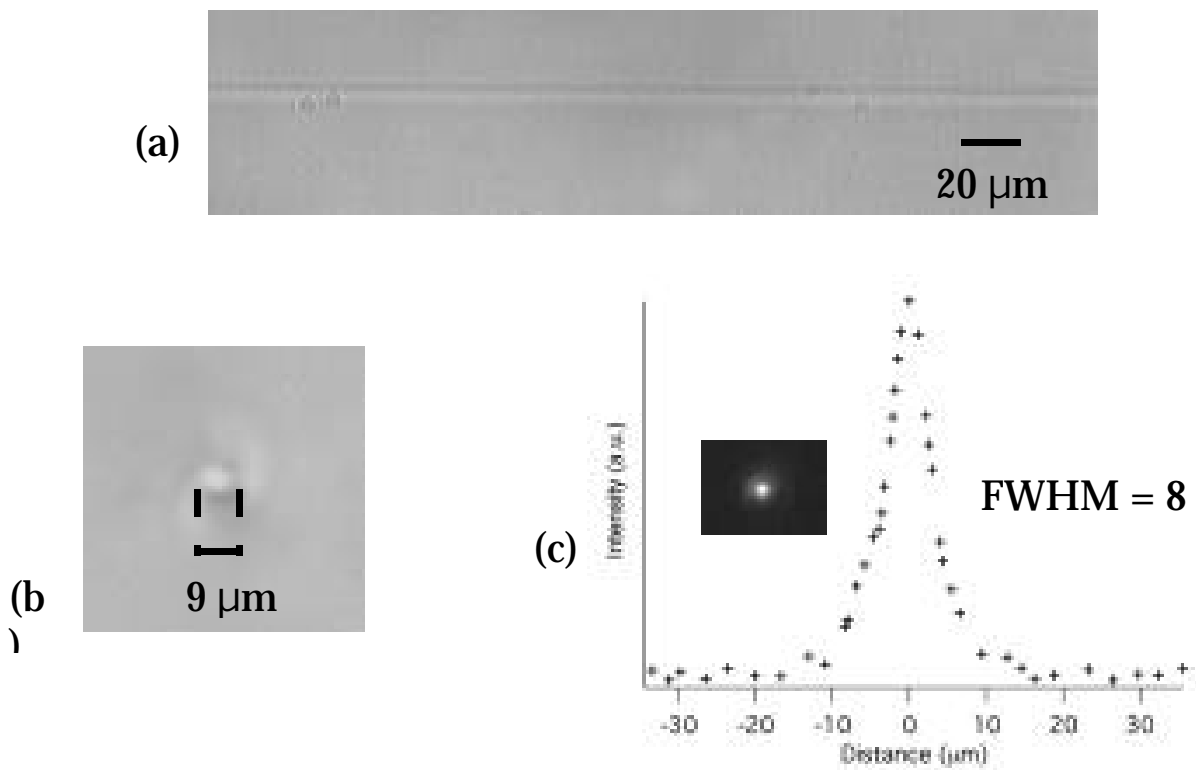

Fig. 3.: (a) Waveguide written inside fused silica using $1 \mu \mathrm{J}$ fs pulse energy (b)White light image of the end face of a $5 \mathrm{~mm}$ long waveguide (c) Intensity distribution of the near field pattern (inset) at $633 \mathrm{~nm}$

\subsubsection{Splitters}

We have also fabricated splitters with splitting angles of $1^{\circ}$ and $1.75^{\circ}$. A schematic diagram showing the dimensions of the $1^{\circ}$ splitter and the corresponding near field mode profile of $633 \mathrm{~nm}$ coupled light through the splitter is shown in Figure 4 . The ratio of the intensity in both arms is roughly $1.1: 1$. The $1.75^{\circ}$ splitter has a $100 \mu \mathrm{m}$ maximum separation between the two arms and an intensity ratio of 1.3:1.

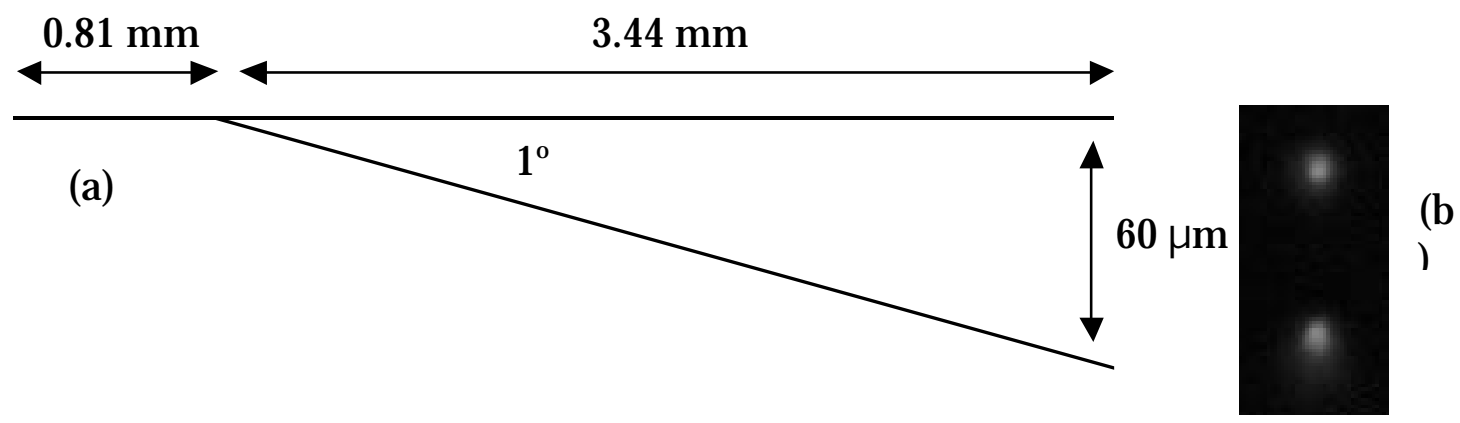


(c)

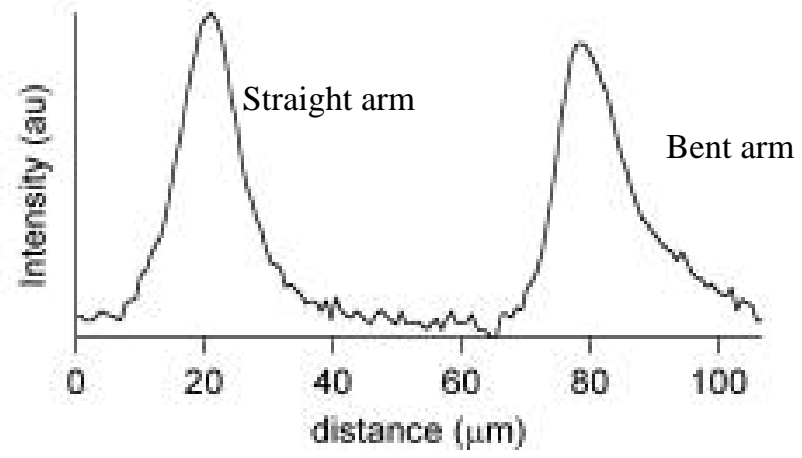

Fig. 4.: (a) Schematic of the $1^{\circ}$ splitter (b) Near field profile of $633 \mathrm{~nm}$ light from the two arms (c) Intensity distribution of the near field pattern

\subsubsection{Mach-Zehnder interferometers}

We have also fabricated Mach-Zehnder interferometers in fused silica using the fs writing technique. The fabrication process required precise motion in the $\mathrm{x}$ - and $\mathrm{y}$ - directions to ensure good overlap of the lines at the two junctions of the two arms. The schematic diagram of the entire interferometer is shown in Figure 5, along with white light microscopic images. The symmetrical interferometer has a splitting angle of $1.5^{\circ}$ and the maximum separation of the two arms is 30 $\mu \mathrm{m}$.

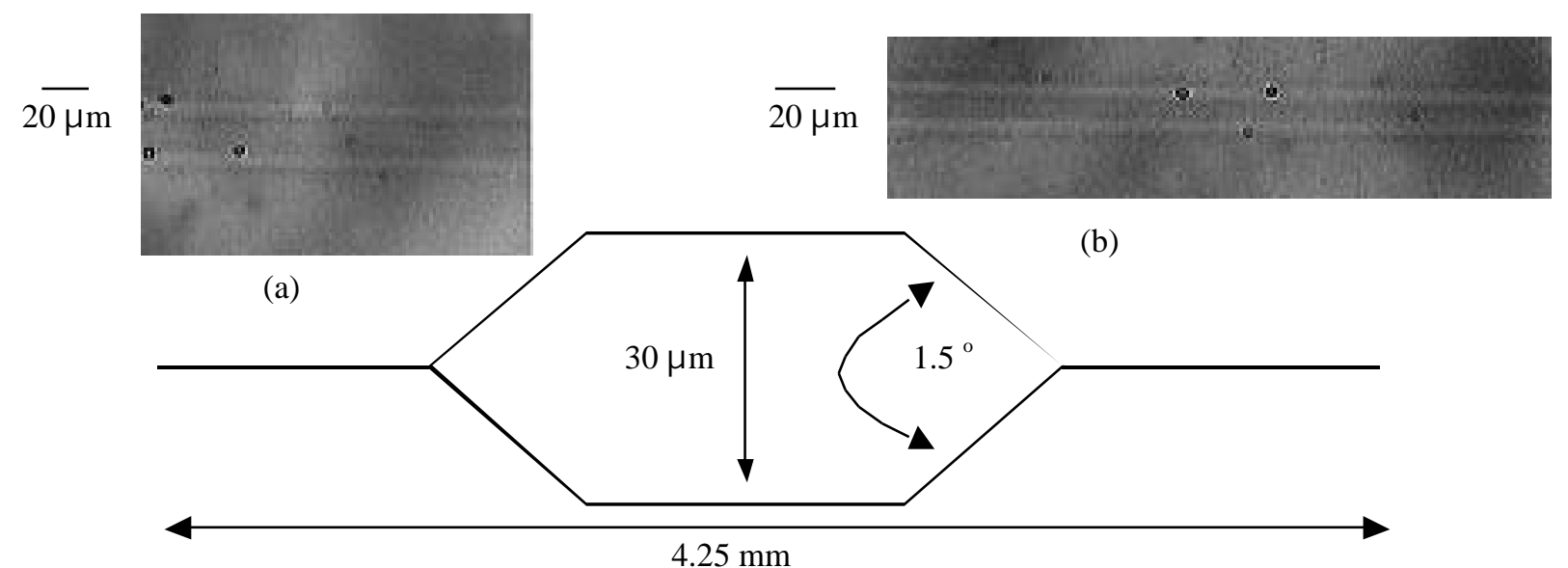

Fig. 5.: Mach-Zehnder interferometer fabricated in fused silica using fs pulses (a) microscope image of the two arms of the interferometer separated by $30 \mu \mathrm{m}$ (b) image of the two arms joining at an angle of $1.5^{\circ}$.

To determine the symmetry of the interferometer, the intensity ratio of $633 \mathrm{~nm}$ coupled light in both arms was first determined by fabricating half of the interferometer, as depicted in Figure 6 and measuring the near field mode intensity of the coupled light. The intensity from both arms is determined to be identical, indicating a splitting ratio of 1:1. 

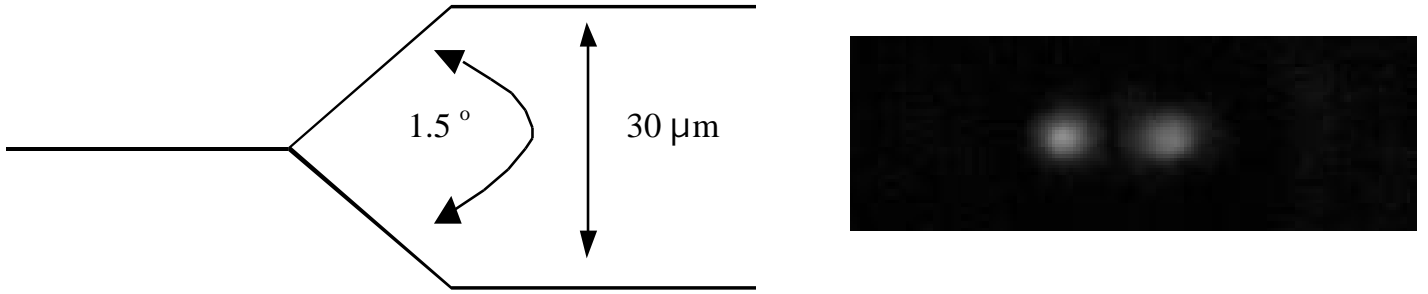

Fig. 6.: Splitting ratio of the symmetrical interferometer is $1: 1$.

He-Ne light at $633 \mathrm{~nm}$ was coupled into the Mach-Zehnder interferometer and the near field pattern exiting the interferometer, shown in Figure 7, has a Gaussian profile with a FWHM of roughly $10 \mu \mathrm{m}$.

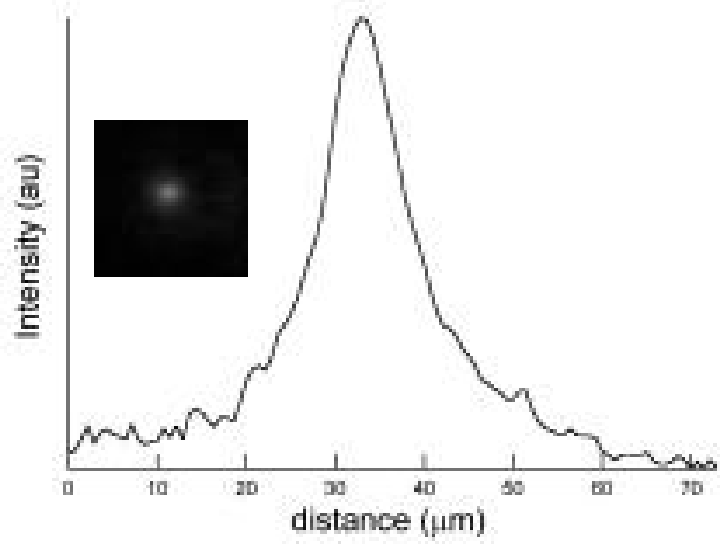

Fig. 7.: Mode profile of $633 \mathrm{~nm}$ light exiting the interferometer.

An issue concerning the fabrication of such complex devices is that the refractive index at the locations in the device where two lines overlap may be higher than at other locations because that location was scanned several times by the fs laser beam. It would be desirable to understand the relationship between the induced index change and such parameters as fs pulse energy and total number of scans by the laser for precise control of device parameters during the fabrication process. The Mach-Zehnder interferometer, which is commonly used as a refractive index sensor, can be used for this purpose. Small changes in the refractive index in one arm induce a phase difference between the $633 \mathrm{~nm}$ light coupled into each arm. This phase difference will result in a change in the output intensity of the coupled light $\left(I_{\text {out }} \approx 1+\cos \delta\right)$. By measuring the output intensity profile, the phase difference and hence, changes in the refractive index can be accurately measured. By modifying one arm of the interferometer with different fs pulse energies or number of scans and measuring the output intensity of $633 \mathrm{~nm}$ coupled light, the refractive index change as a function of the fs writing conditions can be determined.

\subsection{Fluorescence spectroscopy of fs-modified fused silica}

Using a confocal microscopy setup, we have probed the fs-modified fused silica using $488 \mathrm{~nm}$ excitation light. We have observed a broad fluorescence band centered at $630 \mathrm{~nm}$, whose intensity increased with increasing fs pulse energy used 
to modify the glass as shown in Figure 8. The fluorescence comes from non-bridging oxygen hole center (NBOHC) which absorb the $488 \mathrm{~nm}$ light. The fluorescence band has been widely studied and is also observed when fused silica is irradiated with x-rays, neutrons or UV lasers. ${ }^{10}$ A strong fluorescence has also been observed by Sun et al. ${ }^{9}$ after exposure of fused silica to fs laser pulses or after densification by shock waves. The fs laser pulses induce formation of a plasma inside the glass which breaks bonds and produces this color center defect, which consists of a Si-O group with a hole trapped on the oxygen atom. More defects are created as higher fs pulse energies are used. In addition, we also observed that the intensity of the $630 \mathrm{~nm}$ fluorescence decays with prolonged exposure to the low intensity $488 \mathrm{~nm}$ light. A typical result is shown in Figure 9, where $2.0 \mu \mathrm{J}$ fs pulse energy was used to modify the glass. The decay behavior indicates that the color center defects are being photobleached by the $488 \mathrm{~nm}$ light.

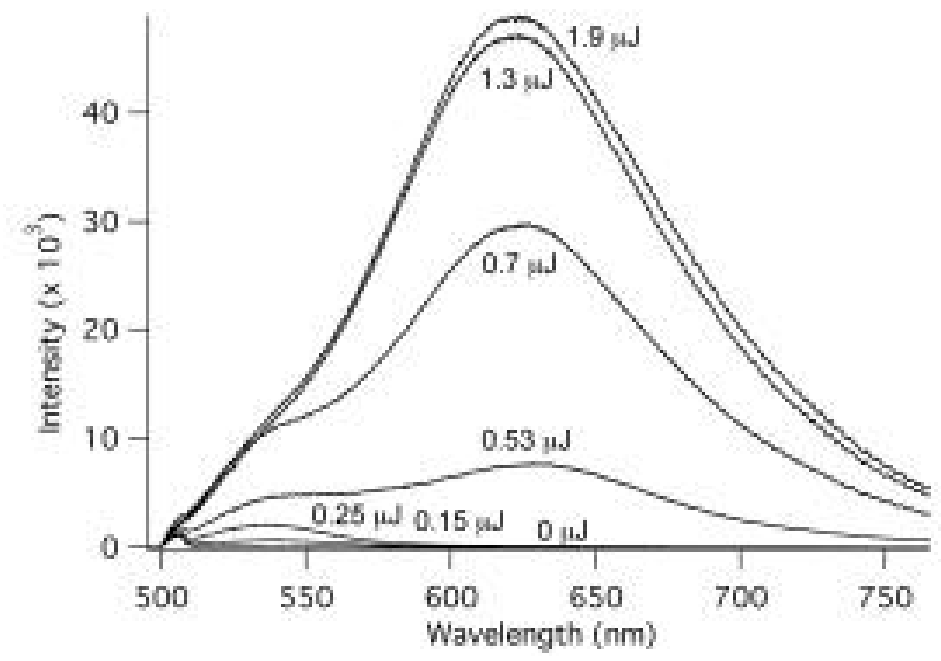

Fig. 8.: Fluorescence band centered at $630 \mathrm{~nm}$ is observed when the modified glass is exposed to $488 \mathrm{~nm}$ laser light. The intensity increases for increasing fs pulse energy used to modify the glass

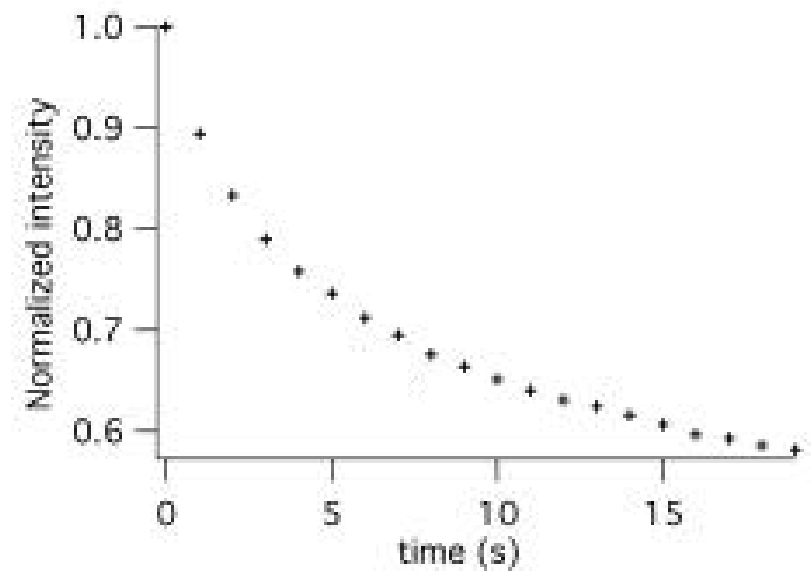

Fig. 9.: Typical decay profile of the fluorescence intensity as a function of exposure time to $10 \mathrm{~mW} 488 \mathrm{~nm}$ $\mathrm{cw}$ laser light. The glass was modified using $2 \mu \mathrm{J}$ of fs pulse energy.

Although it has been shown that densification of the material following fs laser irradiation is a major contributor to the refractive index change of the material, the formation of these color center defects may also contribute to the index change. The defects introduce new absorption states in the material, thus changing the refractive index through the Kramers-Kronig relations. Furthermore, the photobleaching process may have an adverse effect on the stability of the 
index change, since the destruction of the color centers may modify the index change. To understand the role of the color centers and photobleaching process on the index change, we are currently carrying out experiments in which we use the Mach-Zehnder interferometer described in Section 3.1.3 to measure small changes in the refractive index. As one arm of the interferometer is illuminated with the $488 \mathrm{~nm}$ light to photobleach the color centers, any slight changes in the refractive index associated with the photobleaching can be determined by measuring the output intensity (phase difference) of the $633 \mathrm{~nm}$ coupled light.

\section{CONCLUSION}

Waveguides, curved waveguides, splitters, and Mach-Zehnder interferometers have been fabricated inside fused silica by scanning the glass with respect to the focal point of a fs laser beam delivered through a microscope objective.

Fluorescence spectroscopy of the modified material using $488 \mathrm{~nm}$ light has shown a broad fluorescence at $630 \mathrm{~nm}$ which can be attributed to the presence of non-bridging oxygen hole center defects. The color centers are also photobleached by the $488 \mathrm{~nm}$ excitation source. In order to investigate the dependence of the refractive index change on such parameters as fs pulse energy, multiple scans of the fs laser beam, and photobleaching with $488 \mathrm{~nm}$ laser light, we have fabricated a Mach-Zehnder interferometer that can be used as a refractive index sensor to detect small index changes in the glass.

\section{ACKNOWLEDGMENTS}

This work was performed under the auspices of the U.S. Department of Energy by University of California Lawrence Livermore National Laboratory, through the Institute for Laser Science and Applications, under contract No. W-7405Eng-48. J.W. Chan and D.M. Krol acknowledge financial support through National Science Foundation grant ECS 0083087.

\section{REFERENCES}

1. K. M. Davis, K. Miura, N. Sugimoto, and K. Hirao, "Writing waveguides in glass with a femtosecond laser," Opt. Lett. 21, pp. 1729-1731, 1996

2. D. Homoelle, S. Wielandy, A. L. Gaeta, N. F. Borelli, and C. Smith, "Infrared photosensitivity in silica glass exposed to femtosecond laser pulses," Opt. Lett. 24, pp. 1311-1313, 1999

3. A. M. Streltsov and N. F. Borelli, "Fabrication and analysis of a directional coupler written in glass by nanojoule femtosecond laser pulses," Opt. Lett. 26, pp.42-43, 2001

4. E. N. Glezer, M. Milosavljevic, L. Huang, R. J. Finlay, T.-H. Her, J. P. Callan, and E. Mazur, "Three-dimensional optical data storage inside transparent materials," Opt. Lett. 21, pp. 2023-2025, 1996

5. D. Du, X. Liu, G. Korn, J. Squier, and G. Mourou, "Laser-induced breakdown by impact ionization in $\mathrm{SiO}_{2}$ with pulse widths from 7 ns to 150 fs," Appl. Phys. Lett. 64, pp. 3071-3073, 1994

6. A.-C. Tien, S. Backus, H. Kapteyn, M. Murnane, and G. Mourou, "Short-pulse laser damage in transparent materials as a function of pulse duration," Phys. Rev. Lett. 82, pp 3883-3886, 1999

7. J. W. Chan, T. Huser, S. Risbud, and D. M. Krol, "Structural changes in fused silica after exposure to focused femtosecond laser pulses," Opt. Lett. 26, pp. 1726-1728, 2001

8. J. W. Chan, T. Huser, S. Risbud, and D. M. Krol, to be published.

9. H.-B. Sun, S. Juodkazis, M. Watanabe, S. Matsuo, H. Misawa, and J. Nishii, "Generation and recombination of defects in vitreous silica induced by irradiation with a near-infrared femtosecond laser," J. Phys. Chem. B 104, pp. $3450-3455,2000$

10. L. Skuja, "The origin of the intrinsic $1.9 \mathrm{eV}$ luminescence band in glassy $\mathrm{SiO}_{2}$," J. Non-Cryst. Solids 179, pp. 51-69, 1994 